\title{
Cross-sectional and prospective associations of sleep, sedentary and active behaviors with mental health in older people: a compositional data analysis from the Seniors-ENRICA-2 study
}

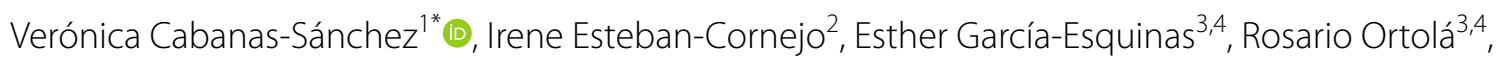
Ignacio Ara ${ }^{5,6}$, Irene Rodríguez-Gómez ${ }^{5,6}$, Sebastien F. M. Chastin ${ }^{7,8}$, Fernando Rodríguez-Artalejo ${ }^{1,3,4}$ and David Martínez-Gómez 1,3,4

\begin{abstract}
Background: Most studies on the effects of sleep, sedentary behavior (SB), and physical activity (PA) on mental health did not account for the intrinsically compositional nature of the time spent in several behaviors. Thus, we examined the cross-sectional and prospective associations of device-measured compositional time in sleep, SB, light PA (LPA) and moderate-to-vigorous PA (MVPA) with depression symptoms, loneliness, happiness, and global mental health in older people ( $\geq 65$ years).

Methods: Data were taken from the Seniors-ENRICA-2 study, with assessments in 2015-2017 (wave 0) and 20182019 (wave 1). Time spent in sleep, SB, LPA and MVPA was assessed by wrist-worn accelerometers. Depression symptoms, loneliness, happiness, and global mental health were self-reported using validated questionnaires. Analyses were performed using a compositional data analysis (CoDA) paradigm and adjusted for potential confounders.

Results: In cross-sectional analyses at wave $0(n=2489)$, time-use composition as a whole was associated with depression and happiness (all $p<0.01$ ). The time spent in MVPA relative to other behaviors was beneficially associated with depression $(\gamma=-0.397, p<0.001)$, loneliness $(\gamma=-0.124, p=0.017)$ and happiness $(\gamma=0.243, p<0.001)$. Hypothetically, replacing 30-min of Sleep, SB or LPA with MVPA was beneficially cross-sectionally related with depression (effect size [ES] ranged -0.326 to -0.246 ), loneliness (ES ranged -0.118 to -0.073 ), and happiness (ES ranged 0.152 to 0.172 ). In prospective analyses $(n=1679)$, MVPA relative to other behaviors at baseline, was associated with favorable changes in global mental health $(\gamma=0.892, p=0.049)$. We observed a beneficial prospective effect on global mental health when 30-min of sleep ( $E S=0.521)$, SB $(E S=0.479)$ or LPA ( $E S=0.755)$ were theoretically replaced for MVPA.
\end{abstract}

Conclusions: MVPA was cross-sectionally related with reduced depression symptoms and loneliness and elevated level of happiness, and prospectively related with enhanced global mental health. Compositional isotemporal analyses showed that hypothetically replacing sleep, SB or LPA with MVPA could result in modest but significantly

*Correspondence: veronica.cabanas@imdea.org

1 IMDEA Food Institute, CEI UAM+CSIC, Madrid, Spain

Full list of author information is available at the end of the article

(c) The Author(s) 2021. Open Access This article is licensed under a Creative Commons Attribution 4.0 International License, which permits use, sharing, adaptation, distribution and reproduction in any medium or format, as long as you give appropriate credit to the original author(s) and the source, provide a link to the Creative Commons licence, and indicate if changes were made. The images or other third party material in this article are included in the article's Creative Commons licence, unless indicated otherwise in a credit line to the material. If material is not included in the article's Creative Commons licence and your intended use is not permitted by statutory regulation or exceeds the permitted use, you will need to obtain permission directly from the copyright holder. To view a copy of this licence, visit http://creativecommons.org/licenses/by/4.0/. The Creative Commons Public Domain Dedication waiver (http://creativeco mmons.org/publicdomain/zero/1.0/) applies to the data made available in this article, unless otherwise stated in a credit line to the data. 
improvements on mental health indicators. Our findings add evidence to the emerging body of research on 24-h time-use and health using CoDA and suggest an integrated role of daily behaviors on mental health in older people.

Keywords: Depression, Loneliness, Happiness, Elderly, Compositional data analysis, Prospective

\section{Background}

The world population is ageing very swiftly [1]. Ageing is a complex process of physical, psychological and social changes that leads to physical decline and increase risk of mental health conditions [2]. The World Health Organization has estimated that approximately $15 \%$ of people aged 60 years and older suffer from a mental disease [1]. Concerns have focused on more severe disorders (e.g., depression) [3], but other relevant mental health conditions, such as loneliness and a low level of happiness, are highly prevalent in old age [4]. Identifying modifiable factors associated with these mental conditions in older adults is essential to promote healthy aging.

There is a large scientific literature about the benefits of physical activity (PA) on mental health in older adults [5]. Also, many studies have addressed the impact of sedentary behavior (SB) and sleep on mental health indicators in this age group [6-9]. Because time is finite during the day (i.e., 24-h), time spent in one behavior is inherently intertwined with time spent in the other activities [10]. Thus, it is important to assess the whole 24-h time-use, and utilize appropriate statistical methods accounting for the co-dependent nature of behaviors. Compositional Data Analysis (CoDA) allows the simultaneous examination of all time-use behaviors by creating a set of log ratios that contain relative information about all behaviors in the 24-h composition and enables accurate interpretation of the behaviors as proportions of total time-use [10,11]. Unfortunately, most previous research failed to account for the compositional nature of time-use; in fact, we are aware of only a few studies, with a cross-sectional design, which used CoDA to investigate the association of time-use composition and mental health in older people [9, 12-15].

Moreover, there is only limited knowledge, proceeding from small number of individuals selected just before the retirement age or from small clinic-based samples, on how time-use composition are longitudinally associated with changes in mental health among older people $[16,17]$. Therefore, using a CoDA approach, we aimed to: (i) examine the cross-sectional associations of accelerometer-derived sleep, SB, light physical activity (LPA) and moderate-to-vigorous physical activity (MVPA) with mental health indicators (i.e., depression symptoms, loneliness, happiness and global mental health) in older people; and (ii) to assess the prospective relationships between time-use composition and changes in mental health indicators in this same population.

\section{Methods \\ Study design and participants}

Data for this study were taken from the SeniorsENRICA-2 cohort, formed by 3,273 community-dwelling individuals aged $\geq 65$ years, enrolled between 2015 and 2017 (wave 0-baseline) in the city of Madrid and four large adjacent cities (Getafe, Torrejón, Alcorcón and Alcalá de Henares) $[18,19]$. In these cities, participants were selected by sex- and district-stratified random sampling of all people holding a national healthcare card (data available at: http://www.comunidad.madrid/ servicios/salud/tarjeta-sanitaria). Among those invited $(n=6418), 51 \%$ accepted to participate in the baseline assessments. Between 2018 and 2019 (wave 1), participants were invited to update the study information. During follow-up, 46 participants died and 1333 were lost, leading to a sample size of 1,894 individuals with wave 0 and wave 1 evaluations.

In the Seniors-ENRICA-2 study, data at both wave 0 and wave 1 were collected in three sequential stages: a) a computer-assisted telephone interview on health status and morbidity, lifestyle habits, and healthcare services use and knowledge; b) a first home visit for performing a complete physical exam, placing accelerometers on the wrist of participants, and collecting blood and urine samples; and, c) a one week-apart second home visit for completing an electronic dietary history and removing the accelerometers.

Study participants and their relatives provided informed written consent, and the study protocol was approved by the Clinical Research Ethics Committee of the La Paz University Hospital, in Madrid. All researchers involved in data collection received appropriate training and were certified before starting the fieldwork.

\section{Assessment of 24-h time-use composition}

Time use composition was determined as the proportions of time spent on sleep, SB, LPA and MVPA objectively assessed by accelerometers. Participants wore an ActiGraph GT9X (ActiGraphInc, Pensacola, FL, USA), attached to the non-dominant wrist using a watch band, for seven consecutive days; they were asked to only 
remove it during water-based activities (i.e., shower, bath or swimming).

The complete protocol for accelerometer data analysis has been detailed elsewhere [18]. Briefly, raw accelerometer data at wave 0 were processed using the GGIR package (v. 1.7-0, https://cran.r-project.org/web/ packages/GGIR/) implemented in R [20]. Sleep periods were detected utilizing an automatized algorithm [18, 21]. Awake time was classified into SB and PA intensities using a previously proposed threshold for ENMO (Euclidean Norm Minus One) in the non-dominant wrist: $<45 \mathrm{mg}$ for $\mathrm{SB}, \geq 45 \mathrm{mg}$ and $<100 \mathrm{mg}$ for LPA, and $\geq 100 \mathrm{mg}$ for MVPA [22]. Days when the accelerometer registered at least $16 \mathrm{~h}$ were considered as valid, and only results from participants with at least 4 valid days (minimum one weekend day) were included in the analyses.

\section{Mental health outcomes}

Depression was ascertained using the 10-item version of the Geriatric Depression Scale (GDS-10) [23]. The following yes/no questions were considered: (1) "Are you basically satisfied with your life?"; (2) "Have you dropped many of your activities and interests?"; (3) "Do you feel that your life is empty?"; (4) "Are you afraid that something bad is going to happen to you?"; (5) "Do you feel happy most of the time?"; (6) "Do you often feel helpless?"; (7) "Do you feel you have more problems with memory than most?"; (8) "Do you feel full of energy?"; (9) "Do you feel that your situation is hopeless?"; and (10) "Do you think that most people are better off than you are?". The negative responses to questions 1,5 and 8 , and the number of positive responses to questions 2,3 , 4, 6, 7, 9 and 10 were summed to calculate a depressive symptoms score (range 0 to 10), with higher scores corresponding to higher levels of depression.

Loneliness was assessed with the three-item loneliness scale [24], which was developed specifically for telephone interviews in large surveys. This scale includes the following three questions: (1) How often do you feel that you lack companionship? (2) How often do you feel left out?, and (3) Hw often do you feel isolated from others?, with response options of hardly ever (coded as 1), some of the time (coded as 2) and often (coded as 3). The sum of three items provides a score ranging from 3 to 9 , with higher scores indicating more loneliness.

Participants reported their level of happiness through the Cantril Ladder of Life Scale [25]. This instrument uses the image of a ladder with steps numbered from zero at the bottom to ten at the top, where the bottom represents the worst possible life and the top the best one. Participants were asked to answer the following question: "On which step of the ladder do you feel you personally stand at the present time?". Thus, score ranged from 0 to 10 with higher values representing a higher happiness state.

Global mental health was assessed using the Spanish version of the 12-item Short Form Health Survey (SF12, version 2) [26]. Participants rated their health, the extent to which their health limited daily activities and their emotional experience during the past 4 weeks, and the mental component summary (MCS) was calculated following the recommendations of the SF-12 developers [26], so that it was standardized to a norm with a mean of 50 and a standard deviation of 10 [27]. Thus, scores ranged from 0 to 100; higher scores in the MCS indicate better mental health, with a 2-point and an 8-point difference deemed to be, respectively, a small and a moderate-to-large difference [27].

These scales were applied at both wave 0 and wave 1 . Changes in each mental health indicator (i.e., depression, loneliness, happiness, and global mental health) were calculated by subtracting the values at wave 0 from the values at wave 1 (e.g., change in depression was calculated as GDS-10 score at wave 1 minus GDS-10 score at wave 0).

\section{Potential confounders}

Self-reported information was collected on sex, age, educational level (less than primary, primary, secondary, or university studies), marital status (single, married, separated, or widowed), household economy (difficult home economy: have great deal of difficulty, difficulty, or some difficulty making ends meet; or easy home economy: have some easy, easy, or great of easy making ends meet), tobacco smoking (current, former or never smoker), and alcohol consume (heavy, moderate, former or never). Food consumption was obtained through a validated computer-assisted face-to-face diet history, and total energy intake ( $\mathrm{kcal} /$ day) was estimated with standard food composition tables [28]. Height and weight were measured by standardized procedures and body mass index (BMI) was calculated as weight $(\mathrm{kg})$ divided by squared height $\left(\mathrm{m}^{2}\right)$. Participants were also asked to report diseases diagnosed by a physician including diabetes mellitus, cardiovascular disease (ischemic heart disease, stroke or heart failure), hypertension, chronic respiratory disease (asthma or chronic bronchitis), osteomuscular disease (osteoarthritis, rheumatoid arthritis or hip fracture), neurodegenerative disease (Parkinson, or dementia/Alzheimer) and cancer at any site. The number of conditions was classified as none, one, and two or more diseases. Cognitive function was assessed with the Mini-Mental State Examination (MMSE) [29], which has been validated for use in Spanish geriatric population [30]. Finally, usual gait speed, a strong marker for physical function in older people, was evaluated by the 8-feet test, following the standardized protocol from the Short 
Physical Performance Battery [31]; gait speed was coded as: (0) unable; $(1) \leq 0.43 \mathrm{~m} / \mathrm{s}$; (2) $0.44-0.60 \mathrm{~m} / \mathrm{s}$; (3) $0.61-$ $0.77 \mathrm{~m} / \mathrm{s} ;(4) \geq 0.78 \mathrm{~m} / \mathrm{s}$.

\section{Statistical analyses}

All analyses and graphical representations were performed with the statistical software R v.3.6.3 (http://cran. rproject.org), using robCompositions package. Statistical significance was set at $p<0.05$. As recently recommended [32], Directed Acyclic Graph (DAG) was developed to completely reflect the relational and causal assumptions of the present study (supplementary Figure 1). DAG was created based on the compositional causal inference framework proposed by Arnold et al. [33] and recent relevant research on physical activity [3-5, 34, 35].

Data were analyzed following the principles of CoDA, proposed by Chastin et al. [10]. The compositional mean was computed calculating the geometric mean for each behavior, and then normalizing the data to the same constant (in this case, 100) and expressing the parts as proportions of the complete 24-h. The distribution of the time composition was illustrated by a matrix of ternary plots with three behaviors represented at a time. The dispersion of the compositional data set was estimated using the variation matrix that summarizes the variability structure of data by means of the log-ratio variance of all pair-wise behaviors. Values close to zero imply that two parts in the ratio are highly co-dependent. We did not need to address the issue of zeros in compositional data, because all participants spent some time in every timeuse behavior.

In order to correctly treat and interpret the compositional daily-behaviors information, data contained in parts of the composition was firstly expressed relative to the other parts as log ratios [10]. Later, time spent in sleep, SB, LPA and MVPA was transformed into three isometric log ratio (ilr) coordinates, applying the structure of the following equations:

$$
\begin{aligned}
& z_{1}=\sqrt{\frac{3}{4}} \ln \left(\frac{\text { Sleep }}{\sqrt[3]{S B * L P A * M V P A}}\right) \\
& z_{2}=\sqrt{\frac{2}{3}} \ln \left(\frac{S B}{\sqrt[2]{L P A * M V P A}}\right) \\
& z_{3}=\sqrt{\frac{1}{2}} \ln \left(\frac{L P A}{M V P A}\right)
\end{aligned}
$$

For cross-sectional analyses, we fitted regression models using time-use composition (expressed as a set of ilr coordinates) as explanatory variables and the mental health indicators at wave 0 as response variables.
Similarly, for prospective analyses, the set of ilr coordinates of the time-use composition was used as explanatory variables, while the change in mental health indicators was considered as dependent variables in regression models. Three models were created with progressive adjustment for potential confounders. Model 1 was adjusted for sex and age; model 2 was additionally adjusted for educational level, marital status, and household economy; and model 3 was further adjusted for smoking status, alcohol consume, total energy intake, BMI, cognitive function (MMSE score), physical function (gait speed test score), and the number of chronic diseases. In prospective analyses, regression models were additionally adjusting for baseline values of each mental health indicator, as appropriate.

Compositional isotemporal substitution analyses, using the above explained regression models, were applied to predict the expected change in outcomes when a fixed duration of time (i.e., $30 \mathrm{~min}$ ) was reallocated from one behavior to another one, while the time in the remaining behaviors was kept constant [36]. The hypothetical change on each mental health indicator (effect size, ES) was estimated for reallocation of time between all possible pair-wise component combinations, in a cross-sectional and prospective approach.

Following the STROBE guidelines [37], a sensitivity analysis was conducted for each model by randomly removing $10 \%$ of the cases and checking for a significant shift in the results; no significant changes were observed. An additional sensitivity analysis was performed analyzing the cross-sectional associations including only participants from the prospective sample. Moreover, since participants with moderate or severe mental impairment might have difficulty self-reporting mental health information, a supplementary sensitivity analysis was executed examining the main cross-sectional and prospective associations after excluding participants with MMSE score $<24$.

\section{Results}

\section{Participants' characteristics}

From the 3,273 participants in the Seniors-ENRICA-2 study, 2,489 provided valid accelerometer data and complete information on potential confounders and, at least, one mental health scale at wave 0 , forming the total analytic sample for this study (cross-sectional sample; Supplementary Figure 2). The sub-sample used for prospective analyses consisted of 1,679 participants with comprehensive data at wave 0 and wave 1 (prospective sample; Supplementary Figure 2). The average time between waves was $2.31 \pm 0.31$ years.

Table 1 shows the main characteristics of the study participants. Briefly, in the cross-sectional sample, the 
Table 1 Descriptive characteristics of the cross-sectional and prospective study sample at wave 0

\begin{tabular}{|c|c|c|c|}
\hline & Cross-sectional sample & Prospective sample & $P$ \\
\hline$n^{a}$ & 2489 & 1679 & \\
\hline Age (years) & $71.68 \pm 4.33$ & $71.40 \pm 4.15$ & 0.054 \\
\hline Sex, n (\%) & & & 0.383 \\
\hline Men & $1168(46.93)$ & $811(48.30)$ & \\
\hline Women & $1321(53.07)$ & $868(51.70)$ & \\
\hline Educational level, n (\%) & & & 0.015 \\
\hline No studies & $384(15.43)$ & $214(12.75)$ & \\
\hline Primary education & $1200(48.21)$ & $792(47.17)$ & \\
\hline Secondary education & $450(18.08)$ & $314(18.70)$ & \\
\hline University or higher education & $455(18.28)$ & $359(21.38)$ & \\
\hline Marital status, n (\%) & & & 0.891 \\
\hline Single & $164(6.59)$ & $114(6.79)$ & \\
\hline Married & $1644(66.05)$ & $1117(66.53)$ & \\
\hline Separated & $173(6.95)$ & $121(7.21)$ & \\
\hline Widowed & $508(20.41)$ & $327(19.48)$ & \\
\hline Household economy, n (\%) & & & 0.093 \\
\hline Difficult economy & $355(14.26)$ & $209(12.45)$ & \\
\hline Easy economy & $2134(85.74)$ & $1470(87.55)$ & \\
\hline Smoking status, n (\%) & & & 0.766 \\
\hline Current & $233(9.36)$ & $154(9.17)$ & \\
\hline Former & $949(38.13)$ & $659(39.25)$ & \\
\hline Never & $1307(52.51)$ & $866(51.58)$ & \\
\hline Alcohol consume, n (\%) & & & 0.706 \\
\hline Heavy drinker ${ }^{b}$ & $116(4.66)$ & $86(5.12)$ & \\
\hline Moderate drinker & $1737(69.79)$ & $1188(70.76)$ & \\
\hline Former & $167(6.71)$ & $106(6.31)$ & \\
\hline Never & $469(18.84)$ & $299(17.81)$ & \\
\hline Total energy intake (kcal/day) & $1944.99 \pm 345.03$ & $1955.57 \pm 344.49$ & 0.331 \\
\hline Body Mass Index $\left(\mathrm{kg} / \mathrm{m}^{2}\right)$ & $27.76 \pm 4.42$ & $27.70 \pm 4.36$ & 0.691 \\
\hline Chronic diseases, n (\%) & & & 0.308 \\
\hline No conditions & $489(19.65)$ & $359(21.38)$ & \\
\hline One condition & $960(38.57)$ & $650(38.71)$ & \\
\hline Two or more conditions & $1040(41.78)$ & $670(39.90)$ & \\
\hline Cognitive function (MMSE) & $27.99 \pm 2.00$ & $28.20 \pm 1.68$ & $<0.001$ \\
\hline Physical function - gait speed test score, n (\%) & & & 0.412 \\
\hline Score 0 (unable) & $7(0.28)$ & $2(0.12)$ & \\
\hline Score $1(\leq 0.43 \mathrm{~m} / \mathrm{s})$ & $90(3.62)$ & $47(2.80)$ & \\
\hline Score $2(0.44-0.60 \mathrm{~m} / \mathrm{s})$ & $224(9.00)$ & $144(8.58)$ & \\
\hline Score $3(0.61-0.77 \mathrm{~m} / \mathrm{s})$ & $437(17.56)$ & $310(18.46)$ & \\
\hline Score 4 ( $\geq 0.78 \mathrm{~m} / \mathrm{s})$ & $1731(69.55)$ & $1176(70.04)$ & \\
\hline Depression score (GDS-10) & $0.93 \pm 1.76$ & $0.80 \pm 1.58^{d}$ & 0.012 \\
\hline Loneliness score (3-items loneliness scale) & $3.69 \pm 1.35$ & $3.68 \pm 1.35^{d}$ & 0.843 \\
\hline Happiness score (0-10) & $7.49 \pm 1.75$ & $7.56 \pm 1.69^{d}$ & 0.196 \\
\hline Global mental health (SF-12) & $50.39 \pm 9.68$ & $50.94 \pm 9.06^{d}$ & 0.067 \\
\hline \multicolumn{4}{|c|}{ Compositional time-use, geometric mean ${ }^{c}(\%, \mathrm{~min} /$ day $)$} \\
\hline Sleep & $32.68(470.59)$ & $32.30(465.12)$ & 0.285 \\
\hline SB & $54.07(778.61)$ & $53.86(775.58)$ & 0.697 \\
\hline LPA & $9.95(143.28)$ & $10.24(147.46)$ & 0.076 \\
\hline MVPA & $3.30(47.52)$ & $3.60(51.84)$ & 0.018 \\
\hline
\end{tabular}


Table 1 (continued)

Values are mean $\pm S D$, unless otherwise indicated

$P=$ differences between cross-sectional and prospective sample, estimated by T-test for continuous variables and Chi-square test for categorical variables

Significant $(p<0.05)$ and borderline $(p<0.1)$ differences are in bold and italics, respectively

Abbreviations: GDS Geriatric Depression Scale, LPA Light Physical Activity, MMSE Mini-Mental State Examination, MVPA Moderate-to-Vigorous Physical Activity, SB Sedentary Behavior, SD Standard Deviation, SF Short Form

${ }^{a} \mathrm{n}$ slightly varies in analyses for each mental health indicator

${ }^{\mathrm{b}}$ Heavy drinker: $\geq 10 \mathrm{~g} /$ day in woman; $\geq 20 \mathrm{~g} /$ day in men

${ }^{c}$ Compositional geometric mean centered to 100 . Values were also expressed as min/day (closed to a constant of $1440 \mathrm{~min} /$ day). Mean of total time registered by accelerometers was $1430.17 \pm 6.52 \mathrm{~min}$ in the cross-sectional sample, and $1430.30 \pm 6.26 \mathrm{~min} /$ day in the prospective sample

${ }^{d}$ Values (mean \pm SD) for mental health indicators at wave 1 for prospective sample was: Depression score: $0.86 \pm 1.57$ ( $p=0.061$ ); Loneliness score: $3.76 \pm 1.40$ $(p=0.006)$; Happiness score: $7.42 \pm 1.63(p<0.001)$; Global mental health: $50.35 \pm 9.69(p=0.016)$. Differences in mental health indicators between wave 0 and wave 1 in prospective sample was estimated by paired T-test

majority of participants were women $(53.07 \%)$, married (66.05\%), never smokers (52.51\%), and moderate drinkers (69.79\%); most had primary educational level (48.21\%), easy home economy (85.74\%), and one chronic disease (38.57\%); the mean total energy intake, BMI and cognitive function score was $1944.99 \mathrm{kcal} /$ day, $27.76 \mathrm{~kg} / \mathrm{m}^{2}$ and 27.99 points, respectively; and most obtained the highest score on the gait speed test (69.55\%). Slight differences between the cross-sectional and prospective sample were found for educational level, cognitive function, depression score, and relative compositional time spent in MVPA.

Ternary diagrams plotting the compositional timeuse distribution at wave 0 for the cross-sectional sample are presented in Fig. 1. Since a four-part composition is examined, the representation is performed in four diagrams containing a 3-dimensional representation of each 3-behavior combination. The vertices of the diagrams represent each behavior of the composition, so that points lying close to a vertex have high percentages of the behavior that is represented by that vertex, while points lying in the center of the triangle have equal percentages of all three behaviors [10]. Likewise, Supplementary Figure 3 illustrate time-use composition for the prospective sample at wave 0 . According to geometric mean of the composition, participants in the cross-sectional sample spent $32.68 \%$ of daily time in sleep (470.59 min/ day), $54.07 \%$ in SB (778.61 $\mathrm{min} /$ day), $9.95 \%$ in LPA (143.28 $\mathrm{min} /$ day), and $3.30 \%$ in MVPA (47.52 min/day; Table 1 ). The variability of the data is summarized by the variation matrix in Supplementary Table 1. The pair-wise log-ratio variances reflected the highest proportional codependence between sleep and SB, while time in MVPA was the least co-dependent on the other behaviors.

\section{Cross-sectional associations of time-use composition with mental health indicators}

In the fully adjusted models for cross-sectional analyses (Table 2), time-use composition as a whole was significantly associated with depression $(p<0.001)$, and happiness $(p<0.001)$, whereas the relation with global mental health remained borderline $(p=0.075)$. The time spent in MVPA relative to other behaviors was beneficially associated with depression $(\gamma=-0.397$, $p<0.001)$, loneliness $(\gamma=-0.124, p=0.017)$ and happiness $(\gamma=0.243, p<0.001)$. Results were similar when crosssectional associations were analyzed including only the participants from the prospective sample (sensitive analysis; Supplementary Table 2).

Table 3 shows the theorical change expected on values of mental health at wave 0 , when 30 -min of time is reallocated between compositional time-use behaviors (fully adjusted models; results for models 1 and 2 are included in Supplementary Table 3). Mainly, replacing 30-min of Sleep, SB or LPA with MVPA was beneficially related with mental health indicators, with effect sizes ranging -0.326 to -0.246 for depression, -0.118 to -0.073 for loneliness, and 0.152 to 0.172 for happiness.

\section{Prospective associations of time-use composition at wave $\mathbf{0}$ with change in mental health indicators}

During the follow-up, depression $(p=0.061)$ and loneliness feelings $(p=0.006)$ increased, while the level of happiness $(p<0.001)$ and global mental health $(p=0.016$; Table 1) decreased. The CoDA models analyzing the association of time-use composition at baseline with changes in mental health indicators over 2.31 years are reported in Table 4. For the fully adjusted models, timeuse distribution as a whole was not significantly associated with the change in mental health indicators (all $\mathrm{p} \geq 0.198$ ). However, the time spent in MVPA relative to other behaviors was associated with favorable changes in global mental health $(\gamma=0.892, p=0.049)$, while sleep time relative to the rest of behaviors was related to adverse changes in depression $(\gamma=0.347, p=0.049)$.

The hypothetical effects of reallocating 30-min between compositional time-use behaviors on the prospective change in mental health indicators are presented in Table 5 (fully adjusted models; results for model 1 and 

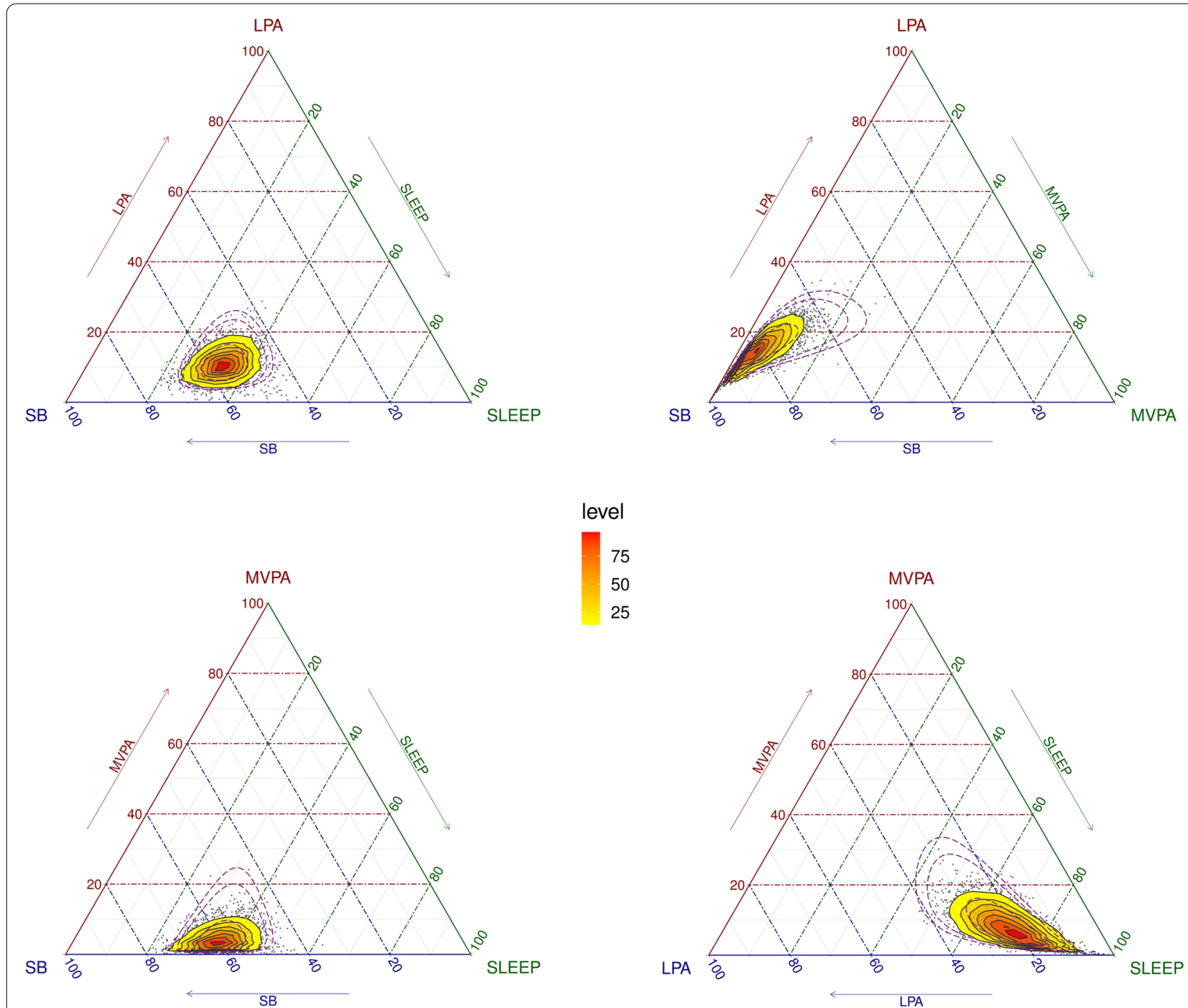

Fig. 1 Ternary plots of the cross-sectional sample compositions of time spent in sleep, sedentary behavior (SB), light physical activity (LPA) and moderate-to-vigorous physical activity (MVPA), at wave 0 . Heat map depicts the frequency distribution of compositions

2 are showed in Supplementary Table 4). We observed a beneficial prospective effect on global mental health when 30 -min of sleep $(E S=0.521)$, $\mathrm{SB}(\mathrm{ES}=0.479)$ or LPA $(E S=0.755)$ were theoretically replaced for MVPA. Substituting SB for sleep was detrimentally related with change on depression $(\mathrm{ES}=0.033)$.

\section{Discussion}

In this cohort of older people, the proportion of time spent in MVPA relative to other behaviors showed the largest associations with better mental health; in particular, MVPA was cross-sectionally related with reduced depression symptoms and loneliness and elevated level of happiness, and prospectively related with enhanced global mental health. Further, compositional isotemporal substitution analyses reveled that theoretically replacing sleep, SB or LPA with MVPA could result in improvements on mental health indicators. Our findings add evidence to the emerging body of research on 24-h time-use and health using CoDA, and suggest an integrated role of daily behaviors on mental health in older people; thus, promoting MVPA, to the detriment of other daily behaviors, could be an effective strategy for encouraging mental health in old age.

Application of CoDA has produced new evidence on the impact of the 24-h behaviors on a wide range of health outcomes $[10,38,39]$. However, only a few previous studies addressed the cross-sectional relationship 
Table 2 Cross-sectional associations between behaviors composition (proportion of the day spent in sleep, SB, LPA and MVPA) and mental health outcomes at wave 0 (cross-sectional sample)

\begin{tabular}{|c|c|c|c|c|c|c|c|c|c|}
\hline & \multirow{2}{*}{$\begin{array}{l}\text { Composition } \\
\text { model } \\
p\end{array}$} & \multicolumn{2}{|l|}{ Sleep } & \multicolumn{2}{|l|}{ SB } & \multicolumn{2}{|l|}{ LPA } & \multicolumn{2}{|l|}{ MVPA } \\
\hline & & $Y$ & $p$ & $\gamma$ & $p$ & $\gamma$ & $p$ & $\gamma$ & $p$ \\
\hline \multicolumn{10}{|c|}{ Depression $(n=2481)$} \\
\hline Model 1 & $<0.001$ & 0.342 & 0.056 & 0.083 & 0.642 & 0.136 & 0.308 & -0.562 & $<0.001$ \\
\hline Model 2 & $<0.001$ & 0.278 & 0.116 & 0.086 & 0.623 & 0.151 & 0.250 & -0.515 & $<0.001$ \\
\hline Model 3 & $<0.001$ & 0.250 & 0.151 & -0.017 & 0.923 & 0.164 & 0.203 & -0.397 & $<0.001$ \\
\hline \multicolumn{10}{|c|}{ Loneliness ( $n=2489)$} \\
\hline Model 1 & 0.007 & 0.056 & 0.696 & -0.008 & 0.958 & 0.113 & 0.279 & -0.162 & 0.003 \\
\hline Model 2 & 0.020 & 0.007 & 0.957 & -0.026 & 0.847 & 0.176 & 0.077 & -0.158 & 0.003 \\
\hline Model 3 & 0.115 & -0.021 & 0.873 & -0.013 & 0.923 & 0.158 & 0.110 & -0.124 & 0.017 \\
\hline \multicolumn{10}{|c|}{ Happiness $(n=2452)$} \\
\hline Model 1 & $<0.001$ & -0.097 & 0.603 & -0.229 & 0.219 & -0.008 & 0.951 & 0.335 & $<0.001$ \\
\hline Model 2 & $<0.001$ & -0.061 & 0.738 & -0.209 & 0.253 & -0.027 & 0.844 & 0.297 & $<0.001$ \\
\hline Model 3 & $<0.001$ & -0.031 & 0.864 & -0.174 & 0.339 & -0.037 & 0.783 & 0.243 & $<0.001$ \\
\hline \multicolumn{10}{|c|}{ Mental health $(n=2443)$} \\
\hline Model 1 & $<0.001$ & -1.418 & 0.172 & 0.235 & 0.817 & -0.027 & 0.972 & 1.209 & 0.002 \\
\hline Model 2 & 0.002 & -0.961 & 0.348 & -0.111 & 0.912 & 0.131 & 0.860 & 0.941 & 0.016 \\
\hline Model 3 & 0.075 & -0.754 & 0.458 & -0.084 & 0.933 & 0.266 & 0.719 & 0.571 & 0.142 \\
\hline
\end{tabular}

Values are shown as non-standardized gamma coefficients $(\gamma)$ from CODA regression models; $\gamma$ can be interpreted as the strength and direction of the association between the amount of time spent in one behavior relative to the others and the mental health outcome

Model 1 adjusted by sex, and age; model 2 adjusted as model 1 plus educational level (no studies, primary, secondary, university), marital status (single, married, separated, widowed), and household economy (difficult economy, easy economy); model 3 adjusted as model 2 plus smoking status (current, former, never), alcohol consume (heavy, moderate, former, never), total energy intake (kcal/day), BMl (kg/m ${ }^{2}$ ), cognitive function (mini-mental state examination score), physical function (gait speed test score: unable, $\leq 0.43 \mathrm{~m} / \mathrm{s}, 0.44-0.60 \mathrm{~m} / \mathrm{s}, 0.61-0.77 \mathrm{~m} / \mathrm{s}, \geq 0.78 \mathrm{~m} / \mathrm{s}$ ), and chronic diseases $(0,1,2+$ )

Statistically significant values are shown in bold $(p<0.05)$ and borderline significant values are shown in italics $(p<0.1)$

Abbreviations: SB Sedentary Behavior, LPA Light Physical Activity, MVPA Moderate-to-Vigorous Physical Activity

between time-use and mental health, utilizing compositional methods $[9,12-15]$. In a research with 430 middle-age participants ( $41.3 \pm 11.7$ years), accelerometer-derived activity composition was associated with physical health-related quality of life (QoL) but not with mental health-related QoL or symptoms of depression, anxiety or stress [12]. Further, two recent studies explored the cross-sectional relationship between $24-\mathrm{h}$ movement behaviors (self-reported sleep duration, and accelerometer-based SB, LPA and MVPA) and mental health among 1095 Japanese workers (50.2 \pm 9.5 years) [14] and 370 Swedish office workers (41 \pm 9 years) [15], using CoDA procedures; in both, the relative time engaged in MVPA was not significantly associated with mental health outcomes. However, in our study, MVPA relative to the other behaviors showed a beneficial cross-sectional association with depression symptoms, loneliness feelings and happiness score. Notably, the previous studies included middle-age population, while ours focused on older people. It is plausible that the cross-sectional relationship of daily activity composition and mental health was stronger among the elderly, because in the Canadian Health Measure Survey, an association between higher proportions of devicemeasured MVPA and better mental health was found among those aged $\geq 65$ years but not $18-64$ years [9].

Significant results emerged in prospective analyses were limited, but we found MVPA, relative to the other behaviors, positively associated with changes in global mental health. The comparison with former reports is complicated because only two previous studies using CoDA have investigated the prospective association between daily behaviors and changes in mental health among older people, and showed discordant results. Lewthwaite et al. [16] found that the change in the 24-h behavior composition was associated with change in anxiety, but not in depression. However, the study sample only included 95 patients (70.5 \pm 6.8 years) with chronic obstructive pulmonary disease, which limits the generalizability of results to the general older population. Conversely, Olds et al. [17] reported that changes in the time-use composition following retirement were related to conditional changes in depression, stress and self-esteem, but not to anxiety, well-being or life satisfaction, in 105 participants from the LAW (Life After Work) cohort. These two studies included small sample sizes 
Table 3 Compositional isotemporal substitution analyses showing the hypothetical change on mental health indicators at wave 0 when 30-min of time is re-allocated between compositional behaviors

\begin{tabular}{|c|c|c|c|c|}
\hline & $\begin{array}{l}\text { Depression }(n=2481) \\
\text { ES }(95 \% \mathrm{Cl})\end{array}$ & $\begin{array}{l}\text { Loneliness }(n=2489) \\
\text { ES }(95 \% \mathrm{Cl})\end{array}$ & $\begin{array}{l}\text { Happiness }(n=2452) \\
\text { ES }(95 \% \mathrm{Cl})\end{array}$ & $\begin{array}{l}\text { Mental health }(n=2443) \\
\text { ES }(95 \% \mathrm{Cl})\end{array}$ \\
\hline \multicolumn{5}{|l|}{$\uparrow$ Sleep } \\
\hline$\downarrow S B$ & $0.013(-0.018,0.043)$ & $0.000(-0.023,0.023)$ & $0.006(-0.025,0.038)$ & $-0.031(-0.205,0.143)$ \\
\hline$\downarrow$ LPA & $-0.067(-0.130,-0.004)$ & $-0.045(-0.093,0.004)$ & $0.020(-0.046,0.086)$ & $-0.060(-0.421,0.300)$ \\
\hline$\downarrow M V P A$ & $0.507(0.372,0.642)$ & $0.151(0.044,0.257)$ & $-0.307(-0.452,-0.163)$ & $-0.730(-1.505,0.045)$ \\
\hline \multicolumn{5}{|l|}{$\uparrow S B$} \\
\hline$\downarrow$ Sleep & $-0.014(-0.045,0.018)$ & $0.000(-0.024,0.024)$ & $-0.006(-0.038,0.027)$ & $0.034(-0.145,0.212)$ \\
\hline$\downarrow$ LPA & $-0.080(-0.143,-0.017)$ & $-0.045(-0.093,0.004)$ & $0.014(-0.052,0.080)$ & $-0.029(-0.389,0.331)$ \\
\hline$\downarrow M V P A$ & $0.494(0.364,0.624)$ & $0.150(0.047,0.253)$ & $-0.313(-0.453,-0.174)$ & $-0.699(-1.445,0.047)$ \\
\hline \multicolumn{5}{|l|}{$\uparrow$ LPA } \\
\hline$\downarrow$ Sleep & $0.051(-0.002,0.105)$ & $0.036(-0.004,0.077)$ & $-0.016(-0.071,0.040)$ & $0.059(-0.246,0.363)$ \\
\hline$\downarrow S B$ & $0.065(0.012,0.117)$ & $0.036(-0.004,0.077)$ & $-0.010(-0.065,0.045)$ & $0.025(-0.274,0.325)$ \\
\hline$\downarrow \mathrm{MVPA}$ & $0.559(0.388,0.730)$ & $0.187(0.052,0.321)$ & $-0.323(-0.506,-0.141)$ & $-0.674(-1.655,0.307)$ \\
\hline \multicolumn{5}{|l|}{$\uparrow M V P A$} \\
\hline$\downarrow$ Sleep & $-0.260(-0.330,-0.189)$ & $-0.074(-0.128,-0.019)$ & $0.152(0.078,0.226)$ & $0.386(-0.019,0.790)$ \\
\hline$\downarrow S B$ & $-0.246(-0.310,-0.182)$ & $-0.073(-0.123,-0.023)$ & $0.158(0.090,0.226)$ & $0.352(-0.016,0.721)$ \\
\hline$\downarrow$ LPA & $-0.326(-0.443,-0.209)$ & $-0.118(-0.209,-0.028)$ & $0.172(0.049,0.295)$ & $0.323(-0.345,0.990)$ \\
\hline
\end{tabular}

Values are estimated modification on mental health indicators at wave 0 (effect size and 95\% Confidence Interval) when 30 min were theoretically re-allocated from one behavior to another one, taking constant the time in the other behaviors

Analyses were adjusted by sex (male, female), age (years), educational level (no studies, primary, secondary, university), marital status (single, married, separated, widowed), household economy (difficult economy, easy economy), smoking status (current, former, never), alcohol consume (heavy, moderate, former, never), total energy intake ( $\mathrm{kcal} / \mathrm{day}), \mathrm{BMI}\left(\mathrm{kg} / \mathrm{m}^{2}\right)$, cognitive function (mini-mental state examination score), physical function (gait speed test score: unable, $\leq 0.43 \mathrm{~m} / \mathrm{s}$, $0.44-0.60 \mathrm{~m} / \mathrm{s}, 0.61-0.77 \mathrm{~m} / \mathrm{s}, \geq 0.78 \mathrm{~m} / \mathrm{s})$, chronic diseases $(0,1,2+)$, and exposure outcome at baseline

Statistically significant values are shown in bold $(p<0.05)$

Abbreviations: ES Effect Size, SB Sedentary Behavior, LPA Light Physical Activity, MVPA Moderate-to-Vigorous Physical Activity

and the use of time was ascertained by a 24-h recall tool (Multimedia Activity Recall for Children and Adults); thereby, ours is the first study using compositional methods to examine how accelerometer-derived daily behaviors prospectively associates with mental health in a relatively large cohort of older people.

Unexpectedly, the proportion of time in SB and LPA showed null relationships with mental health indicators in both cross-sectional and prospective analyses. LPA is the larger contributor to energy expenditure in the older population [40] and previous research based on traditional statistical analyses found stronger associations with mental health for LPA than MVPA [7, 8]. In contrast, former studies using CoDA found MVPA but not LPA to be associated with better mental [9] and cognitive [41] health in the elderly. It is plausible that the effect of LPA depends on the time accumulated in MVPA, so that sufficient levels of MVPA may attenuate associations of LPA with mental health. On the other hand, the literature has showed inconsistent relationships between SB and mental health. For example, some studies emphasized that high levels of device-measured SB increased the risk of depression in older people [42], while others found no association
[43]. It has been suggested that different types of SB have distinct impacts on mental health, so that mentally passive SB (e.g., watching TV) shows detrimental associations, whereas mentally active SB (e.g., reading or internet use) seems to have beneficial or null associations with mental health in adults [44] and older adults $[45,46]$. Finally, we only found a borderline detrimental association between sleep time, relative to other behaviors, with depression symptoms in prospective analyses. To date, the relationship between sleep and depression remains unclear. Several studies indicate that short sleep duration has a higher risk of development and recurrence of depression [47, 48]; however, a meta-analysis by Zhai et al. found a higher risk of depression for long sleep duration [6]. Future research should investigate the possible curvilinear and/or bidirectional association of sleep duration with mental health in older people.

At last, the compositional isotemporal substitution analyses performed in the present study suggested that replacing $30 \mathrm{~min}$ of sleep, $\mathrm{SB}$, or LPA with MVPA is theoretically associated with modest but significant improvements in mental health of older people. The small effect sizes detected are consistent with previous 
Table 4 Prospective associations between behaviors composition (proportion of the day spent in sleep, SB, LPA and MVPA) at wave 0 and mental health indicators at wave 1 (prospective sample)

\begin{tabular}{|c|c|c|c|c|c|c|c|c|c|}
\hline & \multirow{2}{*}{$\begin{array}{l}\text { Composition } \\
\text { model } \\
p\end{array}$} & \multicolumn{2}{|l|}{ Sleep } & \multicolumn{2}{|l|}{ SB } & \multicolumn{2}{|l|}{ LPA } & \multicolumn{2}{|l|}{ MVPA } \\
\hline & & $\gamma$ & $p$ & $Y$ & $p$ & $\gamma$ & $p$ & $\gamma$ & $p$ \\
\hline \multicolumn{10}{|c|}{ Depression $(n=1675)$} \\
\hline Model 1 & 0.160 & 0.305 & 0.088 & -0.274 & 0.115 & 0.080 & 0.532 & -0.111 & 0.105 \\
\hline Model 2 & 0.220 & 0.280 & 0.115 & -0.231 & 0.180 & 0.049 & 0.697 & -0.099 & 0.147 \\
\hline Model 3 & 0.198 & 0.347 & 0.049 & -0.326 & 0.057 & 0.049 & 0.695 & -0.071 & 0.301 \\
\hline \multicolumn{10}{|c|}{ Loneliness $(n=1677)$} \\
\hline Model 1 & 0.467 & 0.035 & 0.819 & -0.061 & 0.679 & 0.121 & 0.273 & -0.094 & 0.111 \\
\hline Model 2 & 0.529 & 0.010 & 0.946 & -0.040 & 0.789 & 0.117 & 0.286 & -0.087 & 0.137 \\
\hline Model 3 & 0.518 & 0.008 & 0.956 & -0.036 & 0.808 & 0.116 & 0.289 & -0.088 & 0.133 \\
\hline \multicolumn{10}{|c|}{ Happiness $(n=1649)$} \\
\hline Model 1 & 0.501 & 0.051 & 0.805 & -0.081 & 0.686 & -0.073 & 0.625 & 0.103 & 0.197 \\
\hline Model 2 & 0.681 & 0.026 & 0.899 & -0.023 & 0.907 & -0.094 & 0.523 & 0.092 & 0.247 \\
\hline Model 3 & 0.637 & -0.021 & 0.917 & 0.005 & 0.981 & -0.079 & 0.589 & 0.096 & 0.224 \\
\hline \multicolumn{10}{|c|}{ Mental health $(n=1661)$} \\
\hline Model 1 & 0.140 & -0.587 & 0.625 & 0.500 & 0.668 & -0.947 & 0.271 & 1.034 & 0.025 \\
\hline Model 2 & 0.147 & -0.536 & 0.653 & 0.354 & 0.760 & -0.813 & 0.342 & 0.995 & 0.030 \\
\hline Model 3 & 0.254 & -0.422 & 0.722 & 0.404 & 0.726 & -0.874 & 0.304 & 0.892 & 0.049 \\
\hline
\end{tabular}

Values are shown as non-standardized gamma coefficients $(\gamma)$ from CODA regression models; $\gamma$ can be interpreted as the strength and direction of the association between the time spent in one behavior relative to the others and the change in mental health outcome

Model 1 adjusted by sex (male, female), age (years), and exposure outcome at baseline; model 2 adjusted as model 1 plus educational level (no studies, primary, secondary, university), marital status (single, married, separated, widowed), and household economy (difficult economy, easy economy); model 3 adjusted as model 2 plus smoking status (current, former, never), alcohol consume (heavy, moderate, former, never), total energy intake ( $\mathrm{kcal} / \mathrm{day}), \mathrm{BMI}\left(\mathrm{kg} / \mathrm{m}^{2}\right)$, cognitive function (minimental state examination score), physical function (gait speed test score: unable, $\leq 0.43 \mathrm{~m} / \mathrm{s}, 0.44-0.60 \mathrm{~m} / \mathrm{s}, 0.61-0.77 \mathrm{~m} / \mathrm{s}, \geq 0.78 \mathrm{~m} / \mathrm{s}$ ), and chronic diseases ( $0,1,2+$ ) Statistically significant values are shown in bold $(p<0.05)$ and borderline significant values are shown in italics $(p<0.1)$

Abbreviations: SB Sedentary Behavior, LPA Light Physical Activity, MVPA Moderate-to-Vigorous Physical Activity

estimates using compositional analyses. For example, in a cross-sectional study using a representative sample of 3233 US adults, Pozo et al. reported that reallocating $60 \mathrm{~min}$ from SB to MVPA was associated with significant but small (i.e., -0.09 points) theoretical reductions in depression symptoms [13]. In a longitudinal way, Lewthwaite et al. observed slight favorable changes in anxiety when $30 \mathrm{~min}$ was re-allocated to MVPA replacing $\mathrm{SB}$ (standardized $\mathrm{ES}=0.070$ ) or LPA (standardized $E S=0.056$ ) [16]. It is important to highlight that the cohort used in our study is formed by non-clinical and non-institutionalized population and, therefore, a relatively healthy older population was evaluated. Recent research has advised that the impact of physical activity on mental health indicators may be stronger for clinical populations compared to non-clinical populations [49], which could partly explain the moderate effect sizes reported in the current study. Anyway, given the global burden associated with mental health decline in older people, our results -although showing modest effect sizes-, could be clinically relevant, and suggest that addressing the distribution of daily behaviors (i.e., increasing MVPA) could be an effective approach to promote mental health in older people.

\section{Strengths and limitations}

The major strength of this study was the application of CoDA, which enabled accounting for the codependence between time-used in behaviors, as well as the objective assessment of daily behaviors across 24 -h with accelerometry. Further strengths were the prospective design of the study, the relatively large sample, and the analytical adjustment for a high number of potential confounders. Moreover, linking the compositional time-use at baseline to changes in mental health over time (adjusting for baseline values of mental health indicators) reinforces the advantages of the prospective design, intended to establish the temporality of the associations and reducing reverse causation. The limitations of the study include the relatively short follow-up period, and the observational nature of the study, which does not allow for entirely discarding residual confounding. Further, we used validated tests to assess a wide range of mental health constructs, but the diagnoses by a physician were not available. Nevertheless, to address the possible difficulty of participants with 
Table 5 Compositional isotemporal substitution analyses projecting the theorical effect of reallocating 30-min of time between compositional behaviors on mental health indicators at wave 1

\begin{tabular}{|c|c|c|c|c|}
\hline & $\begin{array}{l}\text { Depression }(n=1675) \\
\text { ES }(95 \% \mathrm{Cl})\end{array}$ & $\begin{array}{l}\text { Loneliness }(n=1677) \\
\text { ES }(95 \% \mathrm{Cl})\end{array}$ & $\begin{array}{l}\text { Happiness }(n=1649) \\
\text { ES }(95 \% \mathrm{Cl})\end{array}$ & $\begin{array}{l}\text { Mental health }(n=1661) \\
\text { ES }(95 \% \text { Cl) }\end{array}$ \\
\hline \multicolumn{5}{|l|}{$\uparrow$ Sleep } \\
\hline$\downarrow S B$ & $0.033(0.002,0.063)$ & $0.002(-0.024,0.029)$ & $-0.001(-0.037,0.034)$ & $-0.040(-0.246,0.166)$ \\
\hline$\downarrow$ LPA & $0.001(-0.060,0.063)$ & $-0.032(-0.085,0.021)$ & $0.023(-0.048,0.094)$ & $0.236(-0.176,0.648)$ \\
\hline$\downarrow M V P A$ & $0.093(-0.030,0.215)$ & $0.093(-0.012,0.198)$ & $-0.101(-0.241,0.039)$ & $-0.962(-1.783,-0.141)$ \\
\hline \multicolumn{5}{|l|}{$\uparrow S B$} \\
\hline$\downarrow$ Sleep & $-0.033(-0.065,-0.002)$ & $-0.002(-0.029,0.025)$ & $0.001(-0.035,0.038)$ & $0.041(-0.170,0.252)$ \\
\hline$\downarrow$ LPA & $-0.031(-0.091,0.030)$ & $-0.034(-0.086,0.019)$ & $0.024(-0.046,0.095)$ & $0.276(-0.133,0.683)$ \\
\hline$\downarrow M V P A$ & $0.061(-0.057,0.178)$ & $0.091(-0.010,0.191)$ & $-0.100(-0.234,0.035)$ & $-0.923(-1.71,-0.136)$ \\
\hline \multicolumn{5}{|l|}{$\uparrow L P A$} \\
\hline$\downarrow$ Sleep & $-0.006(-0.058,0.046)$ & $0.026(-0.019,0.071)$ & $-0.018(-0.079,0.042)$ & $-0.186(-0.537,0.164)$ \\
\hline$\downarrow S B$ & $0.028(-0.023,0.079)$ & $0.028(-0.016,0.072)$ & $-0.020(-0.079,0.039)$ & $-0.228(-0.569,0.114)$ \\
\hline$\downarrow M V P A$ & $0.088(-0.068,0.244)$ & $0.119(-0.015,0.253)$ & $-0.119(-0.299,0.060)$ & $-1.15(-2.197,-0.103)$ \\
\hline \multicolumn{5}{|l|}{$\uparrow M V P A$} \\
\hline$\downarrow$ Sleep & $-0.060(-0.127,0.008)$ & $-0.050(-0.108,0.009)$ & $0.054(-0.024,0.132)$ & $0.521(0.066,0.975)$ \\
\hline$\downarrow S B$ & $-0.026(-0.087,0.036)$ & $-0.047(-0.100,0.006)$ & $0.053(-0.018,0.124)$ & $0.479(0.066,0.892)$ \\
\hline$\downarrow$ LPA & $-0.057(-0.168,0.054)$ & $-0.081(-0.176,0.014)$ & $0.077(-0.051,0.205)$ & $0.755(0.011,1.500)$ \\
\hline
\end{tabular}

Values are estimated modification on mental health indicators at wave 1 (effect size and 95\% Confidence Interval) when 30 min were theoretically re-allocated from one behavior to another one, taking constant the time in the other behaviors

Analyses were adjusted by sex (male, female), age (years), educational level (no studies, primary, secondary, university), marital status (single, married, separated, widowed), household economy (difficult economy, easy economy), smoking status (current, former, never), alcohol consume (heavy, moderate, former, never), total energy intake ( $\mathrm{kcal} / \mathrm{day}), \mathrm{BMI}\left(\mathrm{kg} / \mathrm{m}^{2}\right)$, cognitive function (mini-mental state examination score), physical function (gait speed test score: unable, $\leq 0.43 \mathrm{~m} / \mathrm{s}$, $0.44-0.60 \mathrm{~m} / \mathrm{s}, 0.61-0.77 \mathrm{~m} / \mathrm{s}, \geq 0.78 \mathrm{~m} / \mathrm{s})$, chronic diseases $(0,1,2+)$, and exposure outcome at baseline

Statistically significant values are shown in bold $(p<0.05)$

Abbreviations: ES Effect Size, SB Sedentary Behavior, LPA Light Physical Activity, MVPA Moderate-to-Vigorous Physical Activity

mental impairment in self-report mental health information and to test whether this might affect the results, we rerun the main analyses after excluding participants with an MMSE score $<24$, but we obtained similar estimates (Supplementary Table 5). Finally, our results do not permit to establish the optimal doses of each time-use behavior to maintain or improve mental health in older people.

\section{Conclusion}

The results of the present study showed that timeuse composition was related to mental health in older people. Mainly, time spent in MVPA relative to other behaviors was beneficially associated with depression, loneliness, and happiness in cross-sectional analyses, and with global mental health in prospective analyses. Moreover, compositional isotemporal analyses showed that hypothetically replacing sleep, SB or LPA with MVPA could result in modest but significantly improvements on mental health indicators. Therefore, interventions and recommendations aimed at increasing MVPA in older people could provide beneficial effects on mental health. However, future experimental research should address the identification of effective and realistic strategies to modify daily behavior components from a holistic approach, in order to promote healthy mental aging.

\begin{abstract}
Abbreviations
BMI: Body mass index; CoDA: Compositional Data Analysis; DAG: Directed Acyclic Graph; ENMO: Euclidean Norm Minus One; ES: Effect size; GDS-10: Geriatric Depression Scale - 10 items; ilr: Isometric log-ratio; kcal: Kilocalories; kg: Kilograms; LAW: Life After Work; LPA: Light physical activity; m: Meters; MCS: Mental component summary; MSSE: Mini-Mental State Examination; MVPA: Moderate-to-vigorous physical activity; NHANES: National Health and Nutrition Examination Survey; PA: Physical activity; QoL: Quality of life; SB: Sedentary behavior; SF-12: 12-Item Short Form Health Survey; STROBE: Strengthening the Reporting of Observational studies in Epidemiology; TV: Television.
\end{abstract}

\section{Supplementary Information}

The online version contains supplementary material available at https://doi. org/10.1186/s12966-021-01194-9.

Additional file 1: Supplementary Figure 1. Directed Acyclic Graph (DAG) illustrating the relational and causal assumptions of the present study. *Diagnosed health conditions included in our model were: cardiovascular disease, hypertension, diabetes mellitus, chronic respiratory disease, osteomuscular disease, neurodegenerative disease, and cancer at any site. The covariates node was created to show all confounding variables including in the model that have a potential relationship with both the 24-h composition and the present and future mental health indicators. Some relationships between factors were omitted for clarity. 
Additional file 2: Supplementary Figure 2. Flowchart of the analytical cross-sectional and prospective sample for the present study, from the Seniors-ENRICA-2 participants. ${ }^{\dagger}$ The inclusion criteria were defined as having at least 4 valid days (at least, 1 valid weekend day; a valid day was considered as having at least 16 hours of record). *No significant differences were found between initial sample of Seniors-ENRICA-2 study and cross-sectional sample included in the present study in sex (46.87\% vs. $46.93 \%$ of men, $p=0.965)$ or age (71.85 \pm 4.49 vs. $71.69 \pm 4.33, p=0.161)$.

Additional file 3: Supplementary Figure 3. Ternary plots of the prospective sample compositions of time spent in sleep, sedentary behavior (SB), light physical activity (LPA) and moderate-to-vigorous physical activity (MVPA), at wave 0 . Heat map depicts the frequency distribution of compositions.

Additional file 4: Supplementary Table 1. Compositional variation matrix. Supplementary Table 2. Cross-sectional associations between behaviors composition (proportion of the day spent in sleep, SB, LPA and MVPA) and mental health outcomes at wave 0 (sensitive analyses with prospective sample). Supplementary Table 3. Compositional isotemporal substitution analyses showing the hypothetical change on mental health indicators at wave 0 when 30 -min of time is re-allocated between compositional behaviors (adjusted models 1 and 2). Supplementary

Table 4. Compositional isotemporal substitution analyses projecting the theorical effect of reallocating 30-min of time between compositional behaviors on mental health indicators at wave 1 (adjusted models 1 and 2). Supplementary Table 5. Cross-sectional and prospective associations between behaviors composition (proportion of the day spent in sleep, SB, LPA and MVPA) and mental health outcomes (sensitive analyses excluding participants with middle or severe cognitive impairment -i.e., Mini-Mental State Examination score $<24$ ).

\section{Acknowledgements}

We thank all participants in the Seniors-ENRICA-2 study.

\section{Authors' contributions}

EG-E, FR-A, and DM-G study concept and design. VC-S, IE-C, EG-E, RO, FR-A, and DM-G acquisition of data. VC-S, IE-C, IA, IR-G, SFMC, and DM-G analysis and interpretation of data. VC-S and DM-G drafting of the manuscript. IE-C, EG-E, $R O, I A$, IR-G, SFMC, FR-A, and DM-G critical revision of the manuscript for important intellectual content. VC-S, IE-C, EG-E, RO, IA, IR-G, SFMC, FR-A, and DM-G final approval of the version to be published. VC-S, IE-C, EG-E, RO, IA, IR-G, SFMC, FR-A, and DM-G agreement to be accountable for all aspects of the work.

\section{Funding}

This work was supported by FIS grants 16/609 and 19/319 (State Secretary of $R+D+I$ and FEDER/FSE), MINECO $R+D+I$ grant (DEP2013-47786-R), the SALAMANDER project (PCIN 2016-145), Biomedical Research Networking Center on Frailty and Healthy Aging (CIBERFES) and FEDER funds from the European Union (CB16/10/00477). VC-S is supported by the Spanish Ministry of Science, Innovation and Universities (IJC2018-038008-I). IE-C is supported by the Spanish Ministry of Economy and Competitiveness (RTI2018095284-J-100). DM-G is supported by a'Ramon y Cajal' contract (RYC-201620546). The funders played no role in the study design, the data collection or analysis, the decision to publish, or the preparation of the manuscript.

\section{Availability of data and materials}

The datasets used and/or analyzed during the current study may be available from the Principal Investigator of the project (FR-A) upon reasonable request.

\section{Declarations}

\section{Ethics approval and consent to participate}

The study was approved by the Clinical Research Ethics Committee of the La Paz University Hospital, in Madrid (ref. 07/062833.9/21). Participants and their relatives provided an informed written consent.

\section{Consent for publication}

Not applicable.

\section{Competing interests}

The authors declare none competing interest.

\section{Author details}

${ }^{1}$ IMDEA Food Institute, CEI UAM+CSIC, Madrid, Spain. ${ }^{2}$ PROFITH "PROmoting FITness and Health through physical activity" Research Group, Department of Physical and Sports Education, Faculty of Sport Sciences, University of Granada, Granada, Spain. ${ }^{3}$ Department of Preventive Medicine and Public Health, Universidad Autónoma de Madrid and Idipaz, Madrid, Spain. ${ }^{4} \mathrm{CIBER}$ of Epidemiology and Public Health (CIBERESP), Madrid, Spain. ${ }^{5}$ GENUD Toledo Research Group, Universidad de Castilla-La Mancha, Toledo, Spain. ${ }^{6}$ Biomedical Research Networking Center on Frailty and Healthy Aging (CIBERFES), Madrid, Spain. ${ }^{7}$ School of Health and Life Science, Institute for Applied Health Research, Glasgow Caledonian University, Glasgow, UK. ${ }^{8}$ Department of Movement and Sport Sciences, Ghent Univeristy, Ghent, Belgium.

Received: 12 March 2021 Accepted: 23 August 2021

Published online: 16 September 2021

\section{References}

1. World Health Organization. Mental health and older adults. 2017. Available from: http://www.who.int/mediacentre/factsheets/fs381/en/.

2. Chatterji S, Byles J, Cutler D, Seeman T, Verdes E. Health, functioning, and disability in older adults - present status and future implications. Lancet. 2015;385:563-75

3. Kok RM, Reynolds CF. Management of depression in older adults: a review. JAMA. 2017;317(20):2114-22.

4. Ong AD, Uchino BN, Wethington E. Loneliness and health in older adults: a mini-review and synthesis. Gerontology. 2016;62(4):443-9.

5. Kadariya S, Gautam R, Aro AR. Physical activity, mental health and wellbeing among older adults in South and Southeast Asia: a scoping review. Biomed Res Int. 2019;2019:6752182.

6. Zhai L, Zhang H, Zhang D. Sleep duration and depression among adults: a meta-analysis of prospective studies. Depress Anxiety. 2015;32(9):664-70.

7. Bae W, Ik Suh Y, Ryu J, Heo J. Physical activity levels and well-being in older adults. Psychol Rep. 2017;120(2):192-205.

8. Ku PW, Fox KR, Liao Y, Sun WJ, Chen LJ. Prospective associations of objectively assessed physical activity at different intensities with subjective well-being in older adults. Qual Life Res. 2016;25(11):2909-19.

9. McGregor DE, Carson V, Palarea-Albaladejo J, Dall PM, Tremblay MS, Chastin SFM. Compositional analysis of the associations between 24-h movement behaviours and health indicators among adults and older adults from the Canadian health measure survey. Int J Environ Res Public Health. 2018;15(8):1779.

10. Chastin SFM, Palarea-Albaladejo J, Dontje ML, Skelton DA. Combined effects of time spent in physical activity, sedentary behaviors and sleep on obesity and cardio-metabolic health markers: a novel compositional data analysis approach. PLoS One. 2015;10(10):e0139984.

11. Dumuid D, Stanford TE, Martin-Fernández JA, Pedišić Ž, Maher CA, Lewis LK, et al. Compositional data analysis for physical activity, sedentary time and sleep research. Stat Methods Med Res. 2018;27(12):3726-38.

12. Curtis RG, Dumuid D, Olds T, Plotnikoff R, Vandelanotte C, Ryan J, et al. The association between time-use behaviors and physical and mental wellbeing in adults: a compositional isotemporal substitution analysis. J Phys Act Health. 2020;17(2):197-203.

13. del Pozo CB, Alfonso-Rosa RM, McGregor D, Chastin SF, Palarea-Albaladejo J, del Pozo CJ. Sedentary behaviour is associated with depression symptoms: compositional data analysis from a representative sample of 3233 US adults and older adults assessed with accelerometers. J Affect Disord. 2020;265:59-62.

14. Kitano N, Kai Y, Jindo T, Tsunoda K, Arao T. Compositional data analysis of 24-hour movement behaviors and mental health in workers. Prev Med Rep. 2020;20:101213.

15. Larisch L-M, Kallings LV, Hagströmer M, Desai M, Von Rosen P, Blom V. Associations between $24 \mathrm{~h}$ movement behavior and mental health in office workers. Int J Environ Res Public Health. 2020;17:6214.

16. Lewthwaite H, Olds T, Williams MT, Effing TW, Dumuid D. Use of time in chronic obstructive pulmonary disease: Iongitudinal associations with 
symptoms and quality of life using a compositional analysis approach. PLoS One. 2019;14(3):e0214058.

17. Olds T, Burton NW, Sprod J, Maher C, Ferrar K, Brown WJ, et al. One day you'll wake up and won't have to go to work: the impact of changes in time use on mental health following retirement. PLoS One. 2018;13(6):e019960.

18. Cabanas-Sánchez V, Esteban-Cornejo I, Miqueles JH, Banegas JR, Graciani A, Rodríguez-Artalejo F, et al. Twenty four-hour activity cycle in older adults using wrist-worn accelerometers: the seniors-ENRICA-2 study. Scand J Med Sci Sport. 2020;30(4):700-8.

19. Ortolá R, García-Esquinas E, Cabanas-Sánchez V, Migueles JH, MartínezGómez D, Rodríguez-Artalejo F. Association of physical activity, sedentary behavior and sleep with unhealthy aging: consistent results for devicemeasured and self-reported behaviors using isotemporal substitution models. J Gerontol A Biol Sci Med Sci. 2021;76(1):85-94.

20. van Hees VT, Gorzelniak L, Dean León EC, Eder M, Pias M, Taherian S, et al. Separating movement and gravity components in an acceleration signal and implications for the assessment of human daily physical activity. Müller M, editor. PLoS One. 2013;8(4):e61691.

21. van Hees VT, Sabia S, Jones SE, Wood AR, Anderson KN, Kivimäki M, et al. Estimating sleep parameters using an accelerometer without sleep diary. Sci Rep. 2018:8(1):12975.

22. Hildebrand $M$, Hansen $B H$, van Hees VT, Ekelund U. Evaluation of raw acceleration sedentary thresholds in children and adults. Scand J Med Sci Sports. 2017;27(12):1814-23.

23. Yesavage JA, BrinkTL, Rose TL, Lum O, Huang V, Adey M, et al. Development and validation of a geriatric depression screening scale: a preliminary report. J Psychiatr Res. 1982;17(1):37-49.

24. Hughes ME, Waite LJ, Hawkley LC, Cacioppo JT. A short scale for measuring loneliness in large surveys: results from two population-based studies. Res Aging. 2004;26:655-72.

25. Cantril H. The pattern of human concerns. New Brunswick: Rutgers University Press; 1965

26. Ware JE, Kosinski M, Keller SD. A 12-Item Short-Form Health Survey: construction of scales and preliminary tests of reliability and validity. Med Care. 1996;34(3):220-33.

27. Vilagut G, Valderas JM, Ferrer M, Garin O, López-García E, Alonso J. Interpretación de los cuestionarios de salud SF-36 y SF-12 en España: Componentes físico y mental. Med Clin (Barc). 2008;130(19):726-35.

28. Guallar-Castillón P, Sagardui-Villamor J, Balboa-Castillo T, Sala-Vila A, Astolfi MJA, Pelous MDS, et al. Validity and reproducibility of a Spanish dietary history. PLoS One. 2014;9(1):e86074.

29. Perneczky R, Wagenpfeil S, Komossa K, Grimmer T, Diehl J, Kurz A. Mapping scores onto stages: mini-mental state examination and clinical dementia rating. Am J Geriatr Psychiatry. 2006;14(2):139-44.

30. Lobo A, Saz P, Marcos G, Día JL, de la Cámara C, Ventura T, et al. Revalidation and standardization of the cognition mini-exam (first Spanish version of the Mini-Mental Status Examination) in the general geriatric population. Med Clin. 1999;112(20):767-74.

31. Guralnik JM, Simonsick EM, Ferrucci L, Glynn RJ, Berkman LF, Blazer DG, et al. A short physical performance battery assessing lower extremity function: association with self-reported disability and prediction of mortality and nursing home admission. J Gerontol Med Sci. 1994:49(2):85-94.

32. Nielsen RO, Shrier I, Casals M, Nettel-Aguirre A, Møller M, Bolling C, et al. Statement on methods in sport injury research from the 1st METHODS MATTER Meeting, Copenhagen, 2019. Br J Sports Med. 2020;54(15):941-7.

33. Arnold KF, Berrie L, Tennant PWG, Gilthorpe MS. A causal inference perspective on the analysis of compositional data. Int J Epidemiol. 2020;49(4):1307-13.

34. Chastin S, McGregor D, Palarea-Albaladejo J, Diaz KM, Hagströmer M, Hallal PC, et al. Joint association between accelerometry-measured daily combination of time spent in physical activity, sedentary behaviour and sleep and all-cause mortality: a pooled analysis of six prospective cohorts using compositional analysis. Br J Sports Med. 2021. Available from: https://bjsm.bmj.com/content/early/2021/05/09/bjsports-2020102345. [epub ahead of print]

35. Mammen G, Faulkner G. Physical activity and the prevention of depression: a systematic review of prospective studies. Am J Prev Med. 2013;45(5):649-57.

36. Dumuid D, Pedišić Ž, Stanford TE, Martín-Fernández JA, Hron K, Maher CA et al. The compositional isotemporal substitution model: a method for estimating changes in a health outcome for reallocation of time between sleep, physical activity and sedentary behaviour. Stat Methods Med Res. 2019;28(3):846-57.

37. Von Elm E, Altman DG, Egger M, Pocock SJ, Gøtzsche PC, Vandenbroucke JP. The Strengthening the Reporting of Observational Studies in Epidemiology (STROBE) statement: guidelines for reporting observational studies. Ann Intern Med. 2007;147:573-7. American College of Physicians.

38. von Rosen P, Dohrn IM, Hagströmer M. Association between physical activity and all-cause mortality: a 15-year follow-up using a compositional data analysis. Scand J Med Sci Sport. 2020;30(1):100-7.

39. Dumuid D, Lewis LK, Olds TS, Maher C, Bondarenko C, Norton L. Relationships between older adults' use of time and cardio-respiratory fitness, obesity and cardio-metabolic risk: a compositional isotemporal substitution analysis. Maturitas. 2018;110:104-10.

40. Cabanas-Sánchez V, Higueras-Fresnillo S, de la Cámara MA, EstebanCornejo I, Martinez-Gomez D. 24-hour movement and non-movement behaviors in older adults. The IMPACT65+ study. Med Sci Sport Exerc. 2019;51(4):671-80.

41. Amagasa S, Inoue $\mathrm{S}$, Murayama H, Fujiwara T, Kikuchi H, Fukushima N, et al. Associations of sedentary and physically-active behaviors with cognitive-function decline in community-dwelling older adults: compositional data analysis from the NEIGE study. J Epidemiol. 2020;30(11):503-8.

42. Eriksson M, Nääs S, Berginström N, Nordström P, Hansson P, Nordström A. Sedentary behavior as a potential risk factor for depression among 70-year-olds. J Affect Disord. 2020;263:605-8.

43. Rosenberg DE, Bellettiere J, Gardiner PA, Villarreal VN, Crist K, Kerr J. Independent associations between sedentary behaviors and mental, cognitive, physical, and functional health among older adults in retirement communities. J Gerontol A Biol Sci Med Sci. 2016;71(1):78-83.

44. Hallgren M, Nguyen TTD, Owen N, Stubbs B, Vancampfort D, Lundin A, et al. Cross-sectional and prospective relationships of passive and mentally active sedentary behaviours and physical activity with depression. $\mathrm{Br}$ J Psychiatry. 2020;217(2):413-9.

45. Hamer M, Stamatakis E. Prospective study of sedentary behavior, risk of depression, and cognitive impairment. Med Sci Sports Exerc. 2014;46(4):718-23.

46. Andrade-Gómez E, Martínez-Gómez D, Rodríguez-Artalejo F, GarcíaEsquinas E. Sedentary behaviors, physical activity, and changes in depression and psychological distress symptoms in older adults. Depress Anxiety. 2018;35(9):884-97.

47. Sun Y, Shi L, Bao Y, Sun Y, Shi J, Lu L. The bidirectional relationship between sleep duration and depression in community-dwelling middleaged and elderly individuals: evidence from a longitudinal study. Sleep Med. 2018:52:221-9.

48. Jackowska M, Poole L. Sleep problems, short sleep and a combination of both increase the risk of depressive symptoms in older people: a 6-year follow-up investigation from the English Longitudinal Study of Ageing. Sleep Med. 2017:37:60-5.

49. Rebar AL, Stanton R, Geard D, Short C, Duncan MJ, Vandelanotte C. A meta-meta-analysis of the effect of physical activity on depression and anxiety in non-clinical adult populations. Health Psychol Rev. 2015;9(3):366-78

\section{Publisher's Note}

Springer Nature remains neutral with regard to jurisdictional claims in published maps and institutional affiliations. 